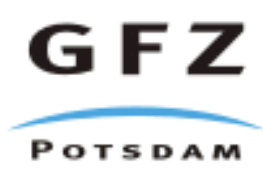

Originally published as:

Wang, R., Lorenzo Martín, F., Roth, F. (2006): PSGRN/PSCMP - a new code for calculating co- and post-seismic deformation, geoid and gravity changes based on the viscoelasticgravitational dislocation theory. - Computers and Geosciences, 32, 4, 527-541

DOI: 10.1016/j.cageo.2005.08.006. 


\title{
PSGRN/PSCMP - a new code for calculating co- and post-seismic deformation, geoid and gravity changes based on the viscoelastic-gravitational dislocation theory
}

\author{
Rongjiang Wang ${ }^{\mathrm{a}, *}$, Francisco Lorenzo Martín ${ }^{\mathrm{b}}$, Frank Roth $^{\mathrm{a}}$ \\ ${ }^{a}$ GeoForschungsZentrum Potsdam (GFZ), Telegrafenberg, D-14473 Potsdam, \\ Germany \\ ${ }^{\mathrm{b}}$ Institute of Geology, Mineralogy and Geophysics, Ruhr University Bochum, \\ D-44780 Bochum, Germany
}

\begin{abstract}
We present a new numerical code for modeling co- and post-seismic response of the Earth's crust to earthquakes. The code consists of two FORTRAN programs: The first program, PSGRN, calculates the time-dependent Green functions of a given layered viscoelastic-gravitational half-space for four fundamental dislocation sources [the strike-slip double-couple, the dip-slip double-couple, the compensated linear vertical dipole (CLVD) and the point inflation] at different depths. The results provide a data base for the second program, PSCMP, which automatically discretizes the earthquake's extended rupture area into a number of discrete point dislocations and calculates the co- and post-seismic deformation by linear superposition. According to the correspondence principle, the same propagator algorithm used in our previously published elastic modeling software, EDGRN/EDCMP, is adopted to compute the spectral Green functions. The temporal Green functions are then obtained by the Fast Fourier Transform (FFT) extended with an anti-aliasing technique, that ensures numerical stability when calculating the post-seismic transients. Moreover, the new software considers the coupling between the deformation and the Earth's gravity field, so that its output includes not only the complete deformation field consisting of 3 displacement components, 6 stress (strain) components and 2 tilt components, but also the geoid and gravity changes. In particular, the gravity effect is treated using a new consistent approach that remedies an incorrect formulation used in many earlier publications. The performance of the software is shown by an example.
\end{abstract}

Key words: Dislocation theory, Laplace-Hankel transform, Green functions, inelastic deformation, the 1960 Valdivia (Chile) earthquake 


\section{Introduction}

During the recent years, revolutionary improvements have been achieved in space geodesy. Especially, since Interferometric Synthetic Aperture Radar (InSAR) and continuous Global Positioning System (GPS) monitoring became more frequently used, not only the instantaneous co-seismic deformation but also delayed post-seismic deformation can be measured with millimeter accuracy (see e.g., Klotz et al., 2001; Jacobs et al., 2002; Fialko, 2004). A possibly important controlling factor for the post-seismic deformation transients is the inelastic response of the Earth, since it consists of an elastic brittle upper crust and ductile viscoelastic deeper parts. The latter might be the asthenosphere, or shallower zones such as, for example, an inelastic lower crust or deeper parts of the fault zone less narrow than the fault itself.

Methods for modeling the elastic co-seismic deformation were developed and widely used in the 1950s and 1960s. Since the late 1960's, three steps were taken to obtain developments for modeling post-seismic effects more comprehensively: (1) the extension to layered half-spaces to treat a medium with an elastic seismogenic part and an inelastic creeping part, (2) viscoelastic behavior was introduced, and (3) gravitation was found to be a non-negligible influence, particularly on the long-term relaxation process for the case of large thrust or normal faulting events, hence it was incorporated.

The first attempts to provide inelastic models were made for the 3D deformation field of an elastic crust above a viscoelastic half-space with different rheologies (Braslau and Lieber, 1968; Rosenman and Singh, 1973a,b; Singh and Rosenman, 1974; Nur and Mavko, 1974). For a review of the progress in these efforts until the late 1990's, see Roth (1994) and Piersanti et al. (1997). Recently, Matsu'ura and Sato (1997) and Hashimoto and Matsu'ura (2000, 2002) included empirical friction laws in the viscoelastic half-space model to explain earthquake cycles by the stress changes along an infinitely long fault due to tectonic loading.

Gravitational effects were first introduced by Rundle (1980a,b, 1981, 1982), with the assumption of depth-independent gravitational acceleration. Between 1994 and 1996, several sequent papers were published that presented the viscoelastic-gravitational dislocation theory and the corresponding numerical tools (see: Fernández and Rundle, 1994a,b; Fernández et al., 1996a,b; Yu et al., 1996a,b). These were followed by similar approaches for the case of magma intrusions (Folch et al., 2000; Fernández et al., 2001), using the source presentation of Bonafede (1990). Recently, Wang (2005a) found an incorrect formulation included in the earlier treatment of the gravity effect, resulting in

\footnotetext{
* Corresponding author (Phone: +49 331 2881209, Fax: +49 331288 1204).

Email address: wang@gfz-potsdam.de (Rongjiang Wang).
} 
new approaches solving the problem being presented by Fernández and Rundle (2004) and Wang (2005b).

According to viscoelasticity theory, post-seismic deformation transients are controlled by (1) the source time function, usually a Heaviside step function, and (2) the relaxation of the shear stress in the inelastic media with time. To avoid the complicated convolution in the time domain, all approaches use the correspondence principle (cf. Fung, 1965), which states that a linear viscoelastic boundary-value problem can be solved by adopting the associated elastic solutions, in which the elastic moduli are replaced by the Laplace or Fourier transformed complex moduli. Then the time-domain solutions are obtained via the inverse Laplace transform. Since the viscoelastic response spectra are usually complicated functions of the Laplace time-conjugate parameter (frequency), results in the closed analytical form are only possible for few particularly simple models, for example the homogeneous viscoelastic half-space without gravity effects. For more complicated models, numerical methods must be used, which in general require a dense sampling of the response spectra that are first obtained by the Haskell propagator algorithm, and then by the Hankel transform. However, both procedures are computationally expensive.

With the increase in computing capacity, various semi-analytical inversion methods were proposed. Schapery (1962) introduced the collocation technique by assuming that the viscoelastic response can be approximated by a finite series of decaying exponentials. Another popular method is the full normal mode analysis introduced by Peltier (1985). Mitrovica and Peltier (1992) compared these two major inversion methods. Their results indicate that the collocation method requires certain preliminary knowledge about the relaxation times. An un-experienced choice of collocation points may lead to erroneous results. In contrast, the full normal mode analysis scans all poles and ensures the inversion accuracy by the residue theorem. However, the finding of poles is very time-consuming, and a complete listing of them is often only possible for a few major relaxation modes for the response functions of selected wavelengths rather than the spatio-temporal Green functions, which generally include an infinite number or even a continuous spectrum of relaxation modes.

With modern computers, the purely numerical inversion is no longer as timeconsuming. The numerical tool presented in this paper is based on the RiemannMellin contour integral in combination with the FFT technique, a straightforward and full-spectrum method for ensuring numerical stability in calculating post-seismic transients. Because of the complicated non-linear relationship between geophysical observables and subsurface structure, a direct inversion for rheological parameters is in general difficult. Since the software provides a forward modeling tool, usually a large set of models must be run to select the best-fitting one. Therefore, the efficiency of the software and the stability of its numerical results are crucial. For this purpose, we present several practical 
techniques used in the new implementation. The efficiency of our computation method is demonstrated by an example.

Here we present a method and software to determine the surface and subsurface deformation, as well as changes in the geoid and gravity, due to the common geophysical sources in a multi-layered viscoelastic-gravitational halfspace. The rheology incorporated is the Standard Linear Solid (SLS) rheology (with the Maxwell rheology as one of its special cases). We have put a strong emphasis on the high performance of the software, both with respect to speed as well as accuracy. This is achieved by the effective techniques (orthonormalized Haskell propagator, analytical asymptotes, filter techniques, etc.) used to solve the stability and convergence problems when computing the Green functions (Wang et al., 2003; R. Wang, 1999; Wang and Kümpel, 2003). These techniques lead to small, fast and very accurate programs.

\section{A consistent approach for including the gravitational effect}

The extension of the earlier elastic dislocation theory with the gravitational effect was first made by Rundle (1980b) based on the generally governing equations for infinitesimal static deformation in a self-gravitating, hydrostatically prestressed Earth (Love, 1911),

$$
\begin{array}{r}
\nabla \cdot \boldsymbol{\Gamma}+\rho \nabla(\psi+\mathbf{u} \cdot \mathbf{g})-\mathbf{g} \nabla \cdot(\rho \mathbf{u})=\mathbf{0} \\
\nabla^{2} \psi-4 \pi G \nabla \cdot(\rho \mathbf{u})=0
\end{array}
$$

where $\boldsymbol{\Gamma}$ is the Lagrangian incremental stress tensor, $\mathbf{u}$ is the displacement vector, $\psi$ is the Eulerian incremental potential, $\mathbf{g}$ is the acceleration due to gravity, $G$ is the gravitational constant, and $\rho$ is the density. For an isotropic and elastic medium, Hooke's linear constitutive relation between the stress and strain holds,

$$
\boldsymbol{\Gamma}=(\lambda \nabla \cdot \mathbf{u}) \mathbf{I}+\mu\left[\nabla \mathbf{u}+(\nabla \mathbf{u})^{T}\right]
$$

where $\lambda$ and $\mu$ are the two Lamé constants, $\mathbf{I}$ is the unit tensor, and $(\nabla \mathbf{u})^{T}$ denotes the tensor transpose of $\nabla \mathbf{u}$.

In order to apply Eqs.(1) and (2) to a simplified plane-Earth model, Rundle approximates the Earth's gravity $\mathbf{g}$ by its surface value and treats it as a constant external body force. Since the self-gravitating term, $\rho \nabla \psi$ in Eq.(1), is negligible for co- and post-seismic deformation, it is in general enough to

consider the so-called reduced problem, in which the deformation equations 
are decoupled from the potential field equation (Rundle, 1982). To construct the Haskell propagator for the reduced problem, four Hankel-transformed fundamental displacement solutions of the poloidal (P-SV) type and two of the toroidal (SH) type are needed. Since the SH solutions include only horizontal movements without volume changes, they are not affected by the gravity field. The Hankel-transformed P-SV solutions with the gravity effect have been given in the form

$$
\left[\begin{array}{c}
U_{z}(k, z) \\
U_{r}(k, z)
\end{array}\right]=\left[\begin{array}{c}
p_{1,2}^{ \pm}(k) \\
1
\end{array}\right] \exp \left[ \pm m_{1,2}(k) z\right]
$$

where $k$ is the horizontal wavenumber (parameter of the Hankel transform), $z$ is the cylindrical coordinate being positive downwards, and $\pm m_{1,2}$ are the four vertical wavenumbers of the deformation field,

$$
\begin{aligned}
& \pm m_{1}= \pm \sqrt{k^{2}+k k_{g}} \\
& \pm m_{2}= \pm \sqrt{k^{2}-k k_{g}}
\end{aligned}
$$

and

$$
p_{1,2}^{ \pm}=\frac{(\lambda+2 \mu) k^{2}-\mu m_{1,2}^{2}}{k\left[\rho g \pm(\lambda+\mu) m_{1,2}\right]}
$$

with

$$
k_{g}=\frac{\rho g}{\sqrt{\mu(\lambda+2 \mu)}} .
$$

For $k>k_{g}$, the elastic-gravitational Haskell propagator can be constructed in analogy to the purely elastic one. Particularly for $k>>k_{g}$, the elasticgravitational solutions converge to the purely elastic ones, implying that the gravity can only affect deformation over long wavelengths.

However, a problem occurs for $k \leq k_{g}$. In this case, the boundary value problem has no solution at all because only one of the four fundamental solutions given by Eq.(4) is regular at infinite depth, but two are in general required. It should be mentioned that the regularity at infinite depth is necessary from both the physical and the mathematical points of view. If any non-regular solution is used instead of the missing regular one, two consequences are expected: (1) A small local perturbation may cause a global reaction of the half-space, and (2) the reaction cannot be uniquely determined because there 
are two non-regular solutions for choice. The parameter $k_{g}$ therefore represents a critical wavenumber of the model. As shown by Wang (2005a), this regularity problem was ignored in many previous studies. In fact, the solution given in Eq.(4) that is valid for $k>k_{g}$ was implicitly extrapolated to the invalid wavenumber region $0 \leq k \leq k_{g}$. Consequently, kernel functions (i.e. the Hankel-transformed response functions) obtained became complex even in the static elastic case and exhibit singularities near the critical wavenumber.

We explain the regularity problem by the physically inconsistent assumptions made for the half-space model. In fact, any compressible medium should become more compact under hydrostatic pressure. Therefore, when including the constant gravity, a self-consistent half-space model should either be incompressible, or have an increasing density with depth. Otherwise, the model is not in a hydrostatical equilibrium state and is therefore unstable. The existence of the critical wavenumber implies that an unpredictable deformation at infinite depth can be induced by a small near-surface perturbation, if the wavelength of the perturbation is large enough - a certainly non-physical consequence.

Another problem is the long-term instability occurring when the shear modulus of a compressible layer of the model relaxes to a very small value. In contrast to the regularity problem for the low wavenumber range, the instability may appear at any wavenumber. However, the physical causes for these two different problems are the same, that is as $\mu \rightarrow 0$, the finite compressible layer becomes practically an infinite medium considering the vanishing gravitational wavelength of this layer $\left(1 / k_{g} \rightarrow 0\right)$.

Wang (2005b) has shown that these two numerical problems can be overcome by using the Adams-Williamson condition (Longman, 1963). This condition requires the density gradient resulting from the initial hydrostatic equilibration. It is given by

$$
\frac{d \rho}{d z}=\frac{\rho^{2} g}{\kappa}
$$

where the parameter $\kappa=\lambda+2 \mu / 3$ is the bulk modulus which is assumed to be constant during the viscoelastic relaxation (see below). In fact, the AdamsWilliamson condition is well satisfied by most realistic Earth models, except for an asthenospheric structure.

When including this density gradient, the equation for the reduced problem reads

$$
\nabla \cdot \Gamma+\rho g \nabla u_{z}-\left(\rho \nabla \cdot \mathbf{u}+\frac{\rho^{2} g}{\kappa} u_{z}\right) g \mathbf{e}_{z}=\mathbf{0}
$$


where $\mathbf{g}=g \mathbf{e}_{z}$ has been used.

In the upper and lower Earth's crust, the density deviations needed to satisfy the Adams-Williamson condition are within a few percent. Therefore, after the density gradient is explicitly considered, the remaining density parameter $\rho$ in Eq.(10) can still be treated as a constant value. Using this approximation, we find that the four vertical wavenumbers given by Eqs.(5) and (6) are modified to

$$
\begin{aligned}
& \pm m_{1}= \pm \sqrt{k^{2}+\frac{2}{3}\left(\tilde{k}_{g}^{2}+\tilde{k}_{g} \sqrt{\tilde{k}_{g}^{2}-k^{2}}\right)} \\
& \pm m_{2}= \pm \sqrt{k^{2}+\frac{2}{3}\left(\tilde{k}_{g}^{2}-\tilde{k}_{g} \sqrt{\tilde{k}_{g}^{2}-k^{2}}\right)}
\end{aligned}
$$

where

$$
\tilde{k}_{g}=\frac{\rho g}{\sqrt{\frac{4}{3} \kappa(\lambda+2 \mu)}} .
$$

The coefficients given by Eq.(7) are still valid.

Note that the real part of the modified eigenvalues $\pm m_{1,2}$ never vanishes for $k>0$. Thus, no critical wavenumber exists even for $\mu \rightarrow 0$. The regularity problem for the low wavenumber range and the numerical instability for the long-term relaxation are therefore avoided by this regularization approach.

\section{$3 \quad$ Implementation of the rheology}

In most seismic reference Earth models, the quality factor of the bulk modulus is at least one order higher than that of the shear modulus. On that account, only the shear modulus will be considered to be viscoelastic while the bulk modulus remains elastic. Viscoelasticity is described by the SLS rheology defined by three parameters: the unrelaxed shear modulus $\mu_{o}$, the viscosity $\eta$ and the parameter $\alpha$ which is the ratio of the fully relaxed modulus to the unrelaxed modulus (Fig. 1). In the frequency domain, the complex shear modulus is then given by

$$
\mu(i \omega)=\mu_{o} \frac{\alpha(1-\alpha)+i \omega \eta / \mu_{o}}{(1-\alpha)+i \omega \eta / \mu_{o}}
$$


where $\omega$ is the angular frequency, and $i=\sqrt{-1}$. Note that the complex shear modulus defined here differs from the Fourier transform of the shear relaxation function by the factor $i \omega$ (see e.g., Christensen, 1971).

Obviously, the SLS rheology becomes identical to the Maxwell rheology when the relaxation ratio $\alpha \rightarrow 0$. Another special case is $\alpha \rightarrow 1$ for the perfect elasticity. For the latter case, the viscosity parameter in Eq.(14) is meaningless.

The complex Lamé constant $\lambda(i \omega)$ can be derived from the complex shear modulus $\mu(i \omega)$ by assuming a constant bulk modulus:

$$
\lambda(i \omega)=\lambda_{o}+\frac{2}{3}\left[\mu_{o}-\mu(i \omega)\right]
$$

where $\lambda_{o}$ represents the unrelaxed Lamé constant.

\section{A practical anti-aliasing technique}

Once the boundary-value problem has been solved in the frequency domain using the correspondence principle, the time-dependent Green functions can be obtained by the inverse Fourier transform. It is known that aliasing problems may appear when using the discrete FFT algorithm.

If the sampling interval of the frequency, which is antiproportional to the time window used, is not high enough, signals beyond the time window will appear at the origin time. These alias signals may result in a wrong estimation of the co-seismic deformation. To solve the problem, we adopt the anti-aliasing technique that has been used in computing synthetic seismograms (Kind and Seidl, 1982). Instead of the Fourier spectrum, for example $X(i \omega)$, the Laplace spectrum $X(\sigma+i \omega)$, where $\sigma$ is a small positive constant, will be computed. The inversion for its time-domain function $x(t)$ is then given by

$$
\begin{aligned}
x(t) & =e^{\sigma t} F^{-1}[X(\sigma+i \omega)] \\
& =e^{\sigma t} \frac{1}{2 \pi} \int_{-\infty}^{+\infty} X(\sigma+i \omega) e^{i \omega t} d \omega .
\end{aligned}
$$

In fact, Eq.(16) can be directly derived from the well-known Riemann-Mellin inversion formula.

Note that $X(\sigma+i \omega)$ is the Fourier spectrum of the function $e^{-\sigma t} x(t)$, i.e. $x(t)$ filtered by the window function $e^{-\sigma t}$. Using Eq. (16), the amplitude of the alias signals appearing at the origin time are reduced by the factor $e^{-\sigma T}$, where $T$ 
is the length of the time window used. We therefore define $\beta=e^{-\sigma T}$ and call it the alias-suppression factor. The smaller the $\beta$ factor chosen, the more effectively are the alias signals suppressed. On the other hand, a too small $\beta$ value will enhance at the same time the numerical errors of the long-term relaxation signals. In most practical cases, to choose $0.1 \leq \beta \geq 0.5$ should be appropriate if the time window used is large enough to cover the main relaxation process.

Another aliasing problem are numerical oscillations due to the limited cut-off frequency used, which is antiproportional to the sampling interval. In general, the viscoelastic response to a Heaviside dislocation source is characterized by three stages: (1) the instantaneous elastic response, (2) the transient viscoelastic relaxation process, and (3) the steady end state. An approximation of such time behavior using a single relaxation time can be expressed in the form

$$
x(t)=\left[x_{p}+\left(x_{i}-x_{p}\right) e^{-t / \tau}\right] H(t),
$$

where $x_{i}$ is the instantaneous co-seismic change, $x_{p}$ is the permanent change, $\tau$ is the relaxation time, and $H(t)$ is the Heaviside function.

The Laplace transform of the function $x(t)$ is

$$
X(\sigma+i \omega)=\frac{x_{p}}{\sigma+i \omega}+\frac{x_{i}-x_{p}}{\sigma+i \omega+1 / \tau}
$$

For a multi-layered model, there may be several relaxation times or even a continuous relaxation spectrum. In this case, the parameter $\tau$ represents the main relaxation time of the model. Because the spectrum given by Eq. (18) converges slowly to zero, numerical oscillations related to the cut-off frequency may appear when using the discrete FFT. To overcome the problem, the coseismic and steady-state solutions, $x_{i}$ and $x_{p}$, respectively, are also computed. Then, Eq. (18) is used for the first-order prediction and subtracted from the computed spectra. The numerical inverse FFT is then only applied to the residual spectra, and the end result is obtained by adding Eq.(17) to the inverse FFT of the residual spectra. The key point of this approach is the estimation of the main relaxation time $\tau$, which may be different for different observables at different positions. In the present implementation, the parameter $\tau$ is estimated independently for each Green function component, following the criterion that the residual takes a value as small as possible at the cut-off frequency.

Numerical tests have shown that the above two techniques are very efficient. The two aliasing problems have been satisfactorily solved. In most cases, a stable FFT result can be obtained even if the time window used is not large enough and/or the time sampling rate is not high enough. 


\section{The computation procedures and the input/output data}

As for our elastic-modeling software, EDGRN/EDCMP, the present viscoelasticgravitational version also consists of two programs. The first program, PSGRN, is used to prepare the time-dependent Green functions that describe the response of the viscoelastic-gravitational model to the 4 fundamental dislocation sources at different depths with a Heaviside time history. The second program, PSCMP, is used to compute the transient deformation, as well as changes in the geoid and gravity field induced by finite fault planes of an earthquake via linear superposition. Usually, PSGRN is considerably more time-consuming than PSCMP. However, once the Green functions have been calculated, they can be repeatedly used for different earthquakes, as long as the Earth model remains unchanged. This is the reason why the computation is divided into these two steps.

Examples for the input files (psgrn.inp for PSGRN and pscmp.inp for PSCMP) are supplied with the programs. They also include explanations of all input parameters. Users can modify the parameter values for their own applications, change the file name, and insert any additional comments.

The output from PSGRN are Green functions covering 13 observables (3 displacement components, 6 stress components, 2 tilt components, and 2 geopotential components, i.e. the gravity and geoid changes) induced by the 4 different dislocation sources (strike-slip, dip-slip, CLVD and inflation). Since the CLVD and inflation sources are axisymmetric, the associated observables are reduced by their tangential components. In summary, there are 44 independent Green functions in the discrete form. The data may then be stored in a given directory to serve as a data base.

As an input of PSCMP, an earthquake is represented by an arbitrary number of rectangular fault planes with different locations and orientations (strike, dip and rake). PSCMP discretizes these fault planes automatically to a set of point dislocations using the same spatial resolution as used for Green functions, and carries out the convolution integration. The observation positions can be either a set of irregular stations, a 1D equidistant profile or a $2 \mathrm{D}$ equidistant array. In addition, users can choose either a local Cartesian coordinate system or the geographic coordinate system. In the latter case, a projection to the local Cartesian system which is needed for the internal convolution procedure will be made using the equality criterion for distance and azimuth. Output from PSCMP are all or a selected part of the 13 observables in the form of time series and/or scenarios ("snapshots"). 


\section{Comparisons with some previously published results}

Wang (2005b) compared the gravity effects on the co- and post-seismic surface deformation computed by the present code and by the code FLTGRV published by Fernández et al. (1996a). In general, both results show that the gravity reduces the magnitude of the long-wavelength part of the vertical displacement of the crust due to the buoyancy effect at the surface and internal density discontinuities. For example, for large thrust earthquakes with a rupture area as large as $400 \times 120 \mathrm{~km}^{2}$, the results of the present code indicate that gravity can affect the co-seismic vertical movement by up to 4 percent. Over longer timescales, when ductile flow in the lower crust must be accounted for, the gravity effect on the vertical displacement can reach up to 20 percent. In comparison, the gravity effect obtained by the code FLTGRV showed a similar spatial form, but seems to include a constant offset, like a rigid-body motion of the crust, in both co- and post-seismic cases. Wang (2005b) concluded that such an offset (comparable with the peak-to-peak amplitude of the gravity effect in the thrust zone.) is unrealistic because it leads to an average surface subsidence, which is inconsistent with thrust-fault mechanisms.

Here we have recomputed deformation models presented by Pollitz (1997). Two Earth models (A and B) were used (see the upper panels of Fig. 2 and 3 ). Figure 2 shows the co- and post-seismic displacement parallel to the fault strike of a strike-slip dislocation. For both models, our results indicate no significant influence of gravity on deformation of strike-slip earthquakes, and agree with those obtained by Pollitz (1997), who used a spherical geometry.

Figure 3 is comparable with Figs. 3 and 6 in Pollitz (1997), showing the vertical displacement and the displacement perpendicular to the fault strike of a buried thrust event. For Model A, we can reproduce Pollitz's results at the co-seismic and early post-seismic period, but find some differences at the late post-seismic period. In particular, for $t=45 \tau$ in the nongravitational case, our results indicate smaller subsidence at the far ends of the half-space profile than those of Pollitz (1997) for the spherical profile. Similar differences can also be seen for Model B. Additionally, our results indicate a smaller gravity effect for Model B than Pollitz's results. All these differences may be attributed to the curvature effect neglected in the present plane-Earth model, but also to the wavelength cut-off (the limited maximum degree of the spherical harmonics) used for the spherical model. It should be mentioned that such a cut-off problem does not exist in the present code, in which the analytical half-space solution (Okada, 1992) is used for the short-wavelength asymptote, so that the numerical calculation is only needed for the long-wavelength residuals due to the gravity effect and the layering of the model. In addition, the analytical propagator scheme used for plane-Earth models is in general more accurate than the numerical propagator scheme (for example the Runge-Kutta 
integration) used for spherical Earth models.

Model A with the same half-space geometry was also considered by Rundle (1982) (see his Figs. 6 and 7). There is a good agreement between his results and ours for the vertical displacement in the nongravitational case, and a slight difference (a few percent) at $t=45 \tau$ in the gravitational case. It seems that numerical errors caused by the previous regularity problem are not so serious for earthquakes with a small to moderate magnitude, but they become non-negligible for large-scale earthquakes (for example $M_{w} \geq 8$ ) as shown by Wang (2005b).

\section{Examples}

As an example of the application of the programs PSGRN/PSCMP, co- and post-seismic deformation induced by the great Valdivia (Chile) earthquake of May 22, $1960\left(M_{w}=9.5\right)$ have been computed. This earthquake is the largest event ever recorded by a seismic network (Kanamori and Cipar, 1974). The strike of the rupture surface was chosen in our model to coincide with the local trend of the Peru-Chile trench, which is about $N 8^{\circ} \mathrm{E}$ (Smith and Sandwell, 1997). The rupture surface had an area of $850 \times 130 \mathrm{~km}^{2}$, dipping $20^{\circ} \mathrm{E}$. The depth of the upper edge of the fault plane was set to $4 \mathrm{~km}$, and a homogeneous slip of $17 \mathrm{~m}$ (Slip distribution 1) is used. The rake angle was fixed at $105^{\circ}$, which coincides with the long-time direction of relative motion between the Nazca and South American plates (Barrientos and Ward, 1990; Plafker and Savage, 1970).

The Earth's crust is modeled by means of a layered half-space, with a $55 \mathrm{~km}$ thick elastic layer over a viscoelastic half-space (Table 1). The viscosity of this underlying half-space is set to $10^{19}$ Pas (Piersanti, 1999; Pollitz et al., 1998). To show the capacity of the software to handle several fault planes, we also consider a second slip distribution (Slip distribution 2). Previous publications suggest that the majority of the slip on the rupture surface of the Valdivia event took place on its northernmost part (Barrientos and Ward, 1990). Accordingly, we also computed the deformation for a model with 3 fault planes, reproducing the same rupture area as before, but assigning a slip of $22 \mathrm{~m}$ to the northernmost $150 \mathrm{~km}, 17 \mathrm{~m}$ to the central $550 \mathrm{~km}$ and $12 \mathrm{~m}$ to the southernmost $150 \mathrm{~km}$.

The results are shown in Figs. 4-8. The dark grey rectangle on each panel shows the projection of the rupture area onto the surface. The upper two panels on each figure show the results for the model with the homogeneous slip distribution, whereas the lower two show the same results for the variable slip distribution on the rupture surface. The two panels on the left of each figure 
reflect the modeled co-seismic deformation associated with the event, the two on the right show to the modeled post-seismic deformation rate 50 years after the event, which roughly corresponds to the present day. Figure 8 shows the time histories of the co- and post-seismic deformation for a position at $73^{\circ} \mathrm{W}$ and $41^{\circ} \mathrm{S}$ and for the two slip models. Interestingly, the N-S displacement, the vertical displacement and the gravity change show non-monotonic variations within the first 100 years following the event, a phenomenon caused by superposition of multiple stress relaxation processes in space and time.

\section{Discussion and conclusions}

In the application example, we modeled the co- and post-seismic deformation of the 1960 Valdivia earthquake for areas up to $500 \mathrm{~km}$ distant from the fault plane. In comparison to spherical models such as those used by, for example Pollitz (1997) and H. Wang (1999), an obvious disadvantage of the plane-Earth model is that it neglects the Earth's surface curvature, which may affect an earthquake's far-field deformation. However, since the source extension of a large earthquake is in general limited to about $1000 \mathrm{~km}$, significant co- and post-seismic deformation may arise at distances of up to a few hundred kilometers from the earthquake's rupture edges. Therefore, the spatial extension of the deformation field, even for the largest earthquake, is smaller than about $1500 \mathrm{~km}$. The maximum arch height of such a spherical area over its average plane surface is less than $25 \mathrm{~km}$, or 2 percent of its horizontal extension. For such a small geometric deviation, we may expect that its influence on the deformation field is similarly small. Thus, the curvature effect should not be larger than a few percent. Additionally, we should keep in mind that the static displacements decrease with distance by about $1 / r^{2}$. Therefore, the co- and post-seismic deformation calculated by PSGRN/PSCMP should be accurate enough for most applications to present-day data sets.

In comparison with other similar modeling tools published previously, the present software PSGRN/PSCMP includes several improvements:

- A new and consistent approach is used for including the gravity effects in the plane-Earth models.

- The loss-of-precision problem of the Haskell propagator algorithm is fully avoided by the orthonormalization technique.

- The numerical accuracy of the inverse Laplace transform is remarkably improved by using FFT with the anti-aliasing extension.

- A data-base of Green functions will be automatically generated, which can be used repeatedly for modeling the deformation field of different earthquakes.

- There is no restriction on the number of layers of the model used. 
- Large-scale earthquakes are represented by a number of rectangular fault planes with different locations and orientations.

- Both geographical and local Cartesian coordinate systems may be used.

- The output includes a complete set of deformation components and the geopotential changes at the surface or any given depth in the form of time series and/or scenarios ("snapshots").

\section{Acknowledgments}

We thank M.E. Belardinelli and an anonymous reviewer for their valuable comments that contributed notably to improving the manuscript. We are grateful to K. Fleming for critical reading and improving English of the revised manuscript. The work was supported by the GeoForschungsZentrum Potsdam (GFZ) and the Deutsche Forschungsgemeinschaft (DFG) under grant SFB 526 (Collaborative Research Centre 'Rheology of the Earth - from the Upper Crust to the Subduction Zone', subproject C5).

\section{References}

Barrientos, S. E., Ward, S. N., 1990. The 1960 Chile earthquake: inversion for slip distribution from surface deformation. Geophysical Journal International 103, 589-598.

Bonafede, M., 1990. Axi-symmetric deformation of a thermo-poro-elastic halfspace: Inflation of a magma chamber. Geophysical Journal International 103, 289-299.

Braslau, D., Lieber, P., 1968. Three-dimensional fields due to a Volterra dislocation embedded in a layered half-space: analytical representation of a seismic mechanism. Bulletin of the Seismological Society of America 58, $613-628$.

Christensen, R. M., 1971. Theory of Viscoelasticity: An Introduction. Academic Press, New York.

Fernández, J., Rundle, J. B., 1994a. Fortran program to compute displacement, potential, and gravity changes resulting from a magma intrusion in a multilayered Earth model. Computers \& Geosciences 20, 461-510.

Fernández, J., Rundle, J. B., 1994b. Gravity changes and deformation due to a magmatic intrusion in a two-layered crustal model. Journal of Geophysical Research 99 (B2), 2737-2746.

Fernández, J., Rundle, J. B., 2004. Postseismic viscoelastic-gravitational half space computations: problems and solutions. Geophysical Research Letters 31, L07608, doi: 10.1029/2004GL019654.

Fernández, J., Tiampo, K. F., Rundle, J. B., 2001. Viscoelastic displacement 
and gravity changes due to point magmatic intrusions in a gravitational layered solid earth. Geophysical Journal International 146 (1), 155-170.

Fernández, J., Yu, T.-T., Rundle, J. B., 1996a. Deformation produced by a rectangular dipping fault in a viscoelastic-gravitational layered Earth model - Part I: Thrust fault FLTGRV and FLTGRH FORTRAN programs. Computers \& Geosciences 22, 735-750, corrections in: Computers \& Geosciences 25 (3), 301-307, 1999.

Fernández, J., Yu, T.-T., Rundle, J. B., 1996b. Horizontal viscoelasticgravitational displacement due to a rectangular dipping thrust fault in a layered Earth model. Journal of Geophysical Research 101, 13,581-13,594, correction in Journal of Geophysical Research 103 (B12), 30,283-6, 1998.

Fialko, Y., 2004. Evidence of fluid-filled upper crust from observations of postseismic deformation due to the $1992 \mathrm{Mw} 7.3$ Landers earthquake. Journal of Geophysical Research 109, doi:10.1029/2003JB002985.

Folch, A., Fernández, J., Rundle, J. B., Martí, J., 2000. Ground deformation in a viscoelastic medium composed of a layer overlying a half-space: a comparison between point and extended sources. Geophysical Journal International 140 (1), 37-50.

Fung, Y. C., 1965. Foundations of Solid Mechanics. Prentice-Hall, 525 pp., Englewood Cliffs, N.J.

Hashimoto, C., Matsu'ura, M., 2000. 3-D physical modelling of stress accumulation processes at transcurrent plate boundaries. Pure and Applied Geophysics 157 (11/12), 2125-2147, spec. iss. on "Microscopic and macroscopic simulation: towards predictive modelling of the earthquake process", ed. by P. Mora, M. Matsu'ura, R. Madariaga, and J.-B. Minster.

Hashimoto, C., Matsu'ura, M., 2002. 3-D simulation of earthquake generation cycles and evolution of fault constitutive properties. Pure and Applied Geophysics 159 (10), 2175-2199, spec. iss. on 'Earthquake processes: physical modelling, numerical simulation and data analysis, part II'; ed. by M. Matsu'ura, P. Mora, A. Donnellan, and X. C. Yin.

Jacobs, A., Sanswell, D., Fialko, Y., Sichoix, L., 2002. The 1999 (Mw 7.1) Hector Mine, California, earthquake: Near-field postseismic deformation from ERS interferometry. Bulletin of the Seismological Society of America 92, 1433-1442.

Kanamori, H., Cipar, J. J., 1974. Focal process of the great Chilean earthquake May 22, 1960. Physics of the Earth and Planetary Interiors 9, 128-136.

Kind, R., Seidl, D., 1982. Analysis of broadband seismograms from the ChilePeru area. Bulletin of the Seismological Society of America 72, 2131-2145.

Klotz, J., Khazaradze, G., Angermann, D., Reigber, C., Perdomo, R., Cifuentes, O., 2001. Earthquake cycle dominates contemporary crustal deformation in Central and Southern Andes. Earth and Planetary Science Letters 193, 437-446.

Longman, I. M., 1963. A Green's function for determining the deformation of the Earth under surface loads II: Computations and numerical results. Journal of Geophysical Research 68, 485-496. 
Love, A. E. H., 1911. Some Problems of Geodynamics. Dover, New York.

Matsu'ura, M., Sato, T., 1997. Loading mechanism and scaling relations of large interplate earthquakes. Tectonophysics 277 (1-3), 189-198.

Mitrovica, J. X., Peltier, W. R., 1992. Constraints on mantle viscosity from relative sea level variations in Hudson Bay. Geophysical Research Letters $142,1185-1188$.

Nur, A., Mavko, G., 1974. Postseismic viscoelastic rebound. Science 183, 204206.

Okada, Y., 1992. Internal deformation due to shear and tensile faults in a half-space. Bulletin of the Seismological Society of America 82, 1018-1040.

Peltier, W. R., 1985. The Lageos constraint on deep mantle viscosity: results from a new normal mode method for the inversion of viscoelastic relaxation spectra. Journal of Geophysical Research 90, 9411-9421.

Piersanti, A., 1999. Postseismic deformation in Chile: Constraints on the asthenospheric viscosity. Geophysical Research Letters 26 (20), 3157-3160.

Piersanti, A., Spada, G., Sabadini, R., 1997. Global postseismic rebound of a viscoelastic Earth: Theory for finite faults and application to the 1964 Alaska earthquake. Journal of Geophysical Research 102, 477-492.

Plafker, G., Savage, J. C., 1970. Mechanism of the Chilean earthquakes of May 21 and 22, 1960. Geological Socity of America Bulletin 81, 1001-1030.

Pollitz, F. F., 1997. Gravitational viscoelastic postseismic relaxation on a layered spherical Earth. Journal of Geophysical Research 102 (B8), 17,92117,941 .

Pollitz, F. F., Bürgmann, R., Romanowicz, B., 1998. Viscosity of oceanic asthenosphere inferred from remote triggering of earthquakes. Science 280, $1245-1249$.

Rosenman, M., Singh, S. J., 1973a. Quasi-static strains and tilts due to faulting in a viscoelastic half-space. Bulletin of the Seismological Society of America 63, 1737-1752.

Rosenman, M., Singh, S. J., 1973b. Stress relaxation in a semi-infinite viscoelastic earth model. Bulletin of the Seismological Society of America 63, 2145-2154.

Roth, F., 1994. New methods using dislocation theory. Proceedings of the 8th Internat. Symp. on Recent Crustal Movements (CRCM '93) of IUGG and IAG, The Local Organizing Committee for the CRCM '93, Kobe, Dec. 6-11, 1993, spec. iss. of Journal of the Geodetic Society of Japan.

Rundle, J. B., 1980a. Numerical evaluation of a static elastic-gravitational deformation of a layered half-space by point couple-sources. Rep., Sandia National Lab., Albuquerque, N.M., SAND 80-2048.

Rundle, J. B., 1980b. Static elastic-gravitational deformation of a layered half space by point couple sources. Journal of Geophysical Research 85, 53555363.

Rundle, J. B., 1981. Vertical displacements from a rectangular fault in layered elastic-gravitational media. Journal of Physics of the Earth 29, 173-186.

Rundle, J. B., 1982. Viscoelastic-gravitational deformation by a rectangular 
thrust fault in a layered Earth. Journal of Geophysical Research 87, 77877796.

Schapery, R. A., 1962. Approximate methods of transform inversion for viscoelastic stress analysis. Proceedings, 4th U.S. National Congress on Applied Mechanics , 1075.

Singh, S. J., Rosenman, M., 1974. Quasi-static deformation of a viscoelastic half-space by a displacement dislocation. Physics of the Earth and Planetary Interiors 8, 87-101.

Smith, W. H. F., Sandwell, D. T., 1997. Global seafloor topography from satellite altimetry and ship depth soundings. Science 277 (5334), 1956-1962.

Wang, H., 1999. Surface vertical displacements, potential perturbations and gravity changes of a viscoelastic earth model induced by internal point dislocations. Geophysical Journal International 137 (2), 429-440.

Wang, R., 1999. A simple orthonormalization method for the stable and efficient computation of Green's functions. Bulletin of the Seismological Society of America 89, 733-741.

Wang, R., 2005a. On the singularity problem of the elastic-gravitational dislocation theory applied to plane-earth models. Geophysical Research Letters 32, L06307, doi: 10.1029/2003GL019358.

Wang, R., 2005b. The dislocation theory: a consistent way for including the gravity effect in (visco)elastic plane-earth models. Geophysical Journal International 161, 191-196, doi: 10.1111/j.1365-246X.2005.02614.x.

Wang, R., Kümpel, H.-J., 2003. Poroelasticity: Efficient modelling of strongly coupled, slow deformation processes in a multilayered half-space. Geophysics 68, 705-717.

Wang, R., Lorenzo Martín, F., Roth, F., 2003. Computation of deformation induced by earthquakes in a multi-layered elastic crust - FORTRAN programs EDGRN/EDCMP. Computers \& Geosciences 29 (2), 195-207.

Yu, T.-T., Rundle, J. B., Fernández, J., 1996a. Deformation produced by a rectangular dipping fault in a viscoelastic-gravitational layered Earth model - Part II: Strike slip fault STRGRV and STRGRH FORTRAN programs. Computers \& Geosciences 22, 751-764, corrigendum in Computers \& Geosciences 28, 89-91, 2002.

Yu, T.-T., Rundle, J. B., Fernández, J., 1996b. Surface deformation due to a strike-slip fault in an elastic gravitational layer overlying a viscoelastic gravitational half-space. Journal of Geophysical Research 101, 3199-3214, correction: Journal of Geophysical Research 104 (B7), 15313-15315, 1999. 


\section{Tables and figures}

Table 1

The 2-layer half-space model used in the application example. The unrelaxed moduli $\lambda_{o}$ and $\mu_{o}$ are given in round brackets to indicate that they are not independent quantities, but derived from the density $\rho$ and seismic velocities $V_{p}$ and $V_{s} . \eta$ is the viscosity and $\alpha$ is the ratio between the relaxed and unrelaxed shear modulus according to the SLS rheology.

\begin{tabular}{ccccccc}
\hline depth & $\rho$ & $V_{p}$ & $V_{s}$ & $\left(\lambda_{o}=\mu_{o}\right)$ & $\eta$ & $\alpha$ \\
{$\left[10^{3} \mathrm{~m}\right]$} & {$\left[10^{3} \mathrm{~kg} / \mathrm{m}^{3}\right]$} & {$\left[10^{3} \mathrm{~m} / \mathrm{s}\right]$} & {$\left[10^{3} \mathrm{~m} / \mathrm{s}\right]$} & {$\left[10^{10} \mathrm{~Pa}\right]$} & {$\left[10^{19} \mathrm{~Pa} \cdot \mathrm{s}\right]$} & {$[-]$} \\
\hline $0-55$ & 2.90 & 6.70 & 3.87 & $(4.33)$ & $\infty$ & 1.0 \\
$55-\infty$ & 3.40 & 8.00 & 4.62 & $(7.25)$ & 1.00 & 0.0 \\
\hline
\end{tabular}




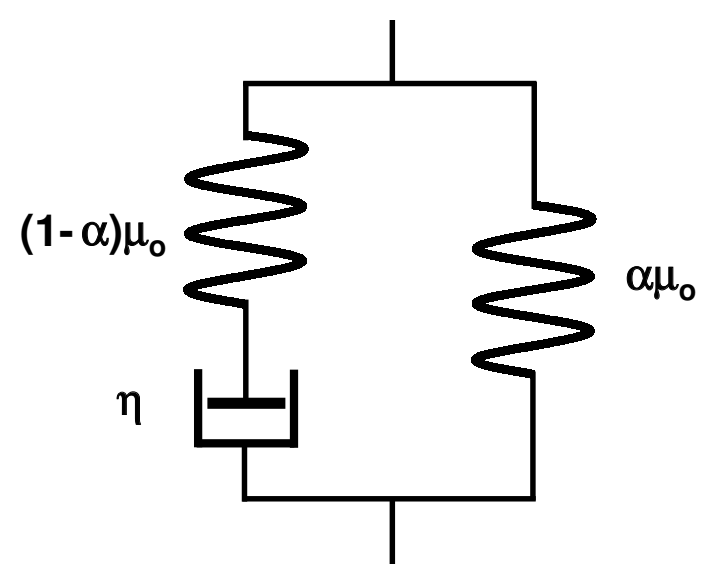

Fig. 1. Model of the SLS rheology. $\mu_{o}$ is the unrelaxed modulus, $\eta$ is the viscosity, and $0 \leq \alpha \leq 1$ is the relaxation ratio. Note the two special cases, $\alpha=1$ and $\alpha=0$, representing perfect elasticity and the Maxwell rheology, respectively. 

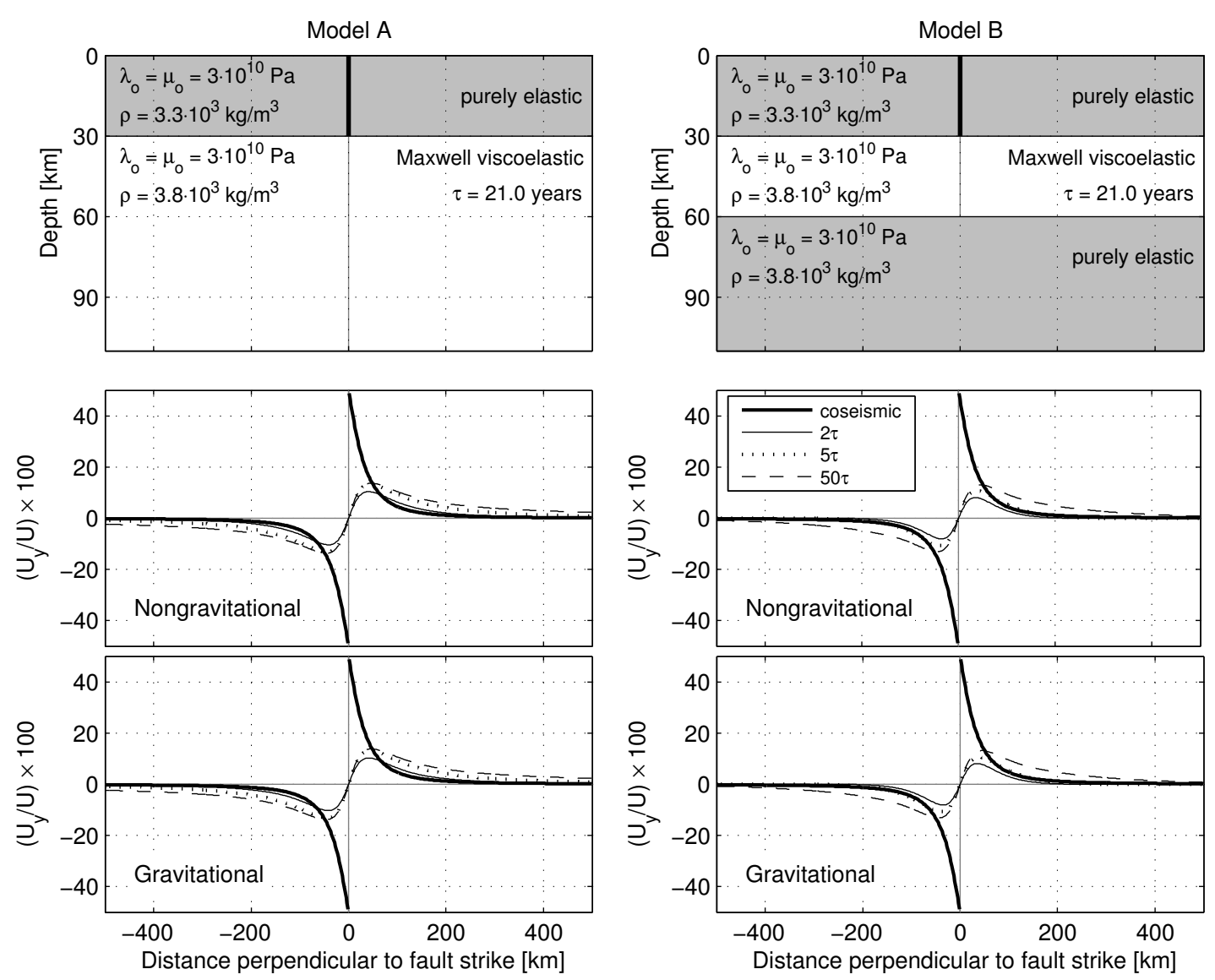

Fig. 2. Coseismic and additional post-seismic surface displacement calculated for two Earth models, for a profile perpendicular to the fault strike through the center of the rupture surface. The source is a vertical strike-slip dislocation. The fault is 200 $\mathrm{km}$ along the strike and $30 \mathrm{~km}$ along the dip (penetrating the entire elastic upper layer). $\tau\left(=2 \eta / \mu_{o}\right)$ is the characteristic Maxwell relaxation time. $U$ represents the magnitude of slip on the fault plane and $U_{y}$ is the displacement parallel to the fault strike. These results are comparable with Figs. 9 and 10 of Pollitz (1997). 

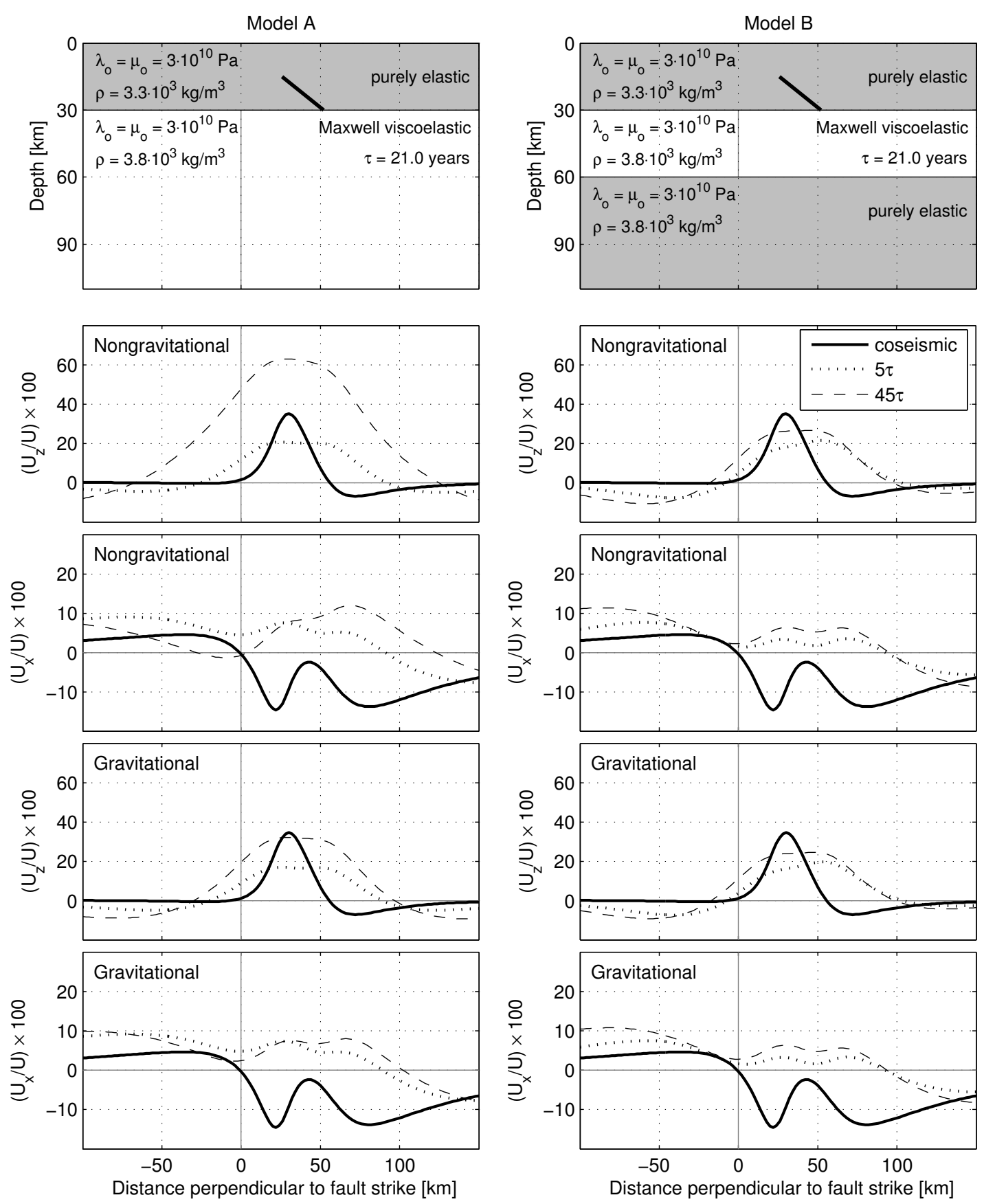

Fig. 3. Same as Fig. 2, but for a buried thrust fault dipping at 30 degree. $U_{x}$ and $U_{z}$ are the displacement perpendicular to the fault strike and the vertical displacement, respectively. These results are comparable with Figs. 3 and 6 of Pollitz (1997) and, for the vertical displacement of Model A, with Figs. 6 and 7 of Rundle (1982). 


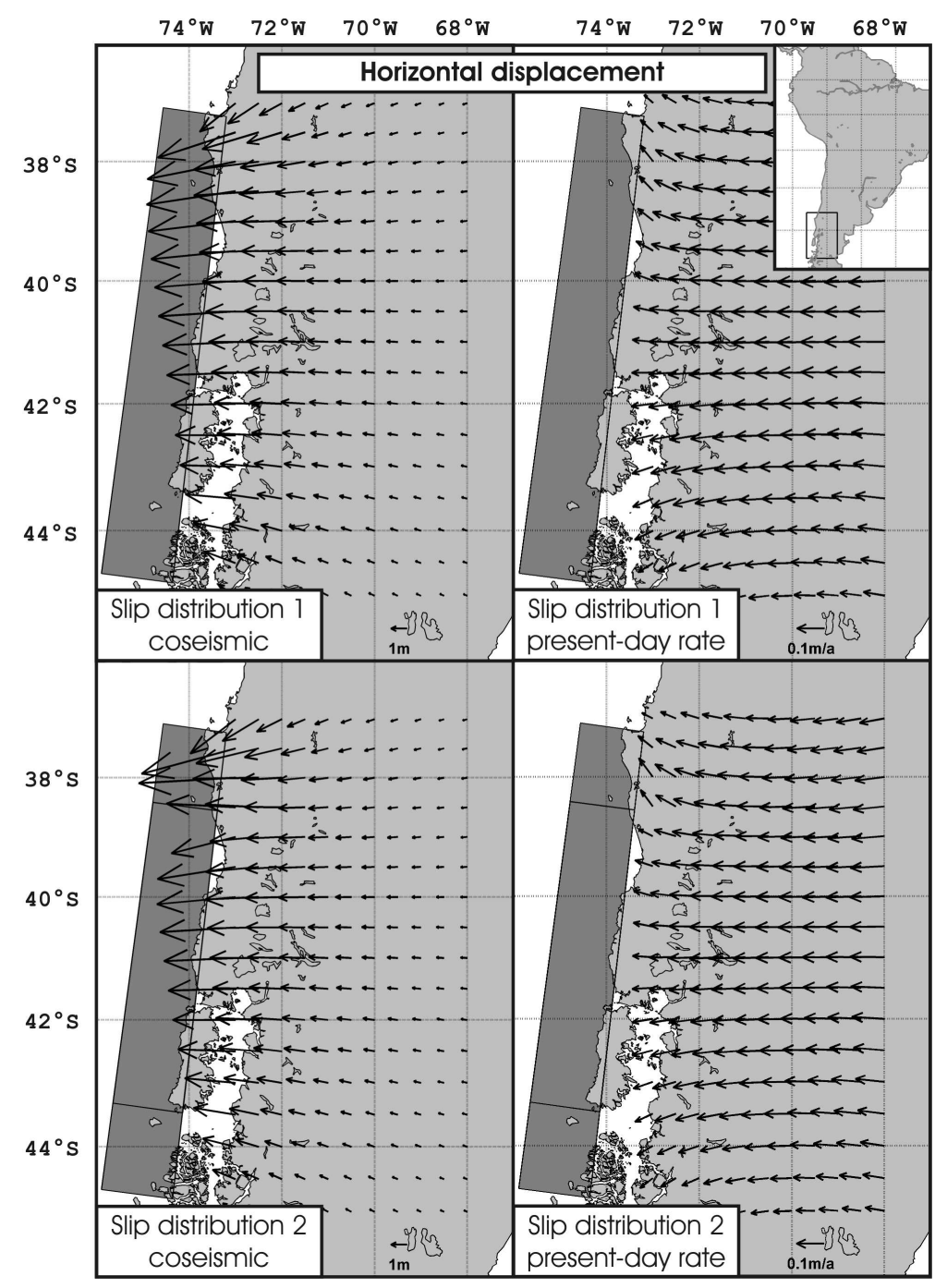

Fig. 4. Horizontal surface displacements and velocities induced by the 1960 Valdivia, Chile, earthquake, computed using PSGRN/PSCMP. The modeled earthquake rupture surface is shown by the dark grey rectangle. The parameters for the fault were chosen from former studies (Barrientos and Ward, 1990; Smith and Sandwell, 1997). Upper two panels show the results for a model with homogeneous slip over the rupture surface. Left: modeled co-seismic deformation; right: modeled post-seismic deformation rate 50 years after the event (present day). Lower two panels show the results for a model with larger slip on the northernmost part of the rupture surface. 


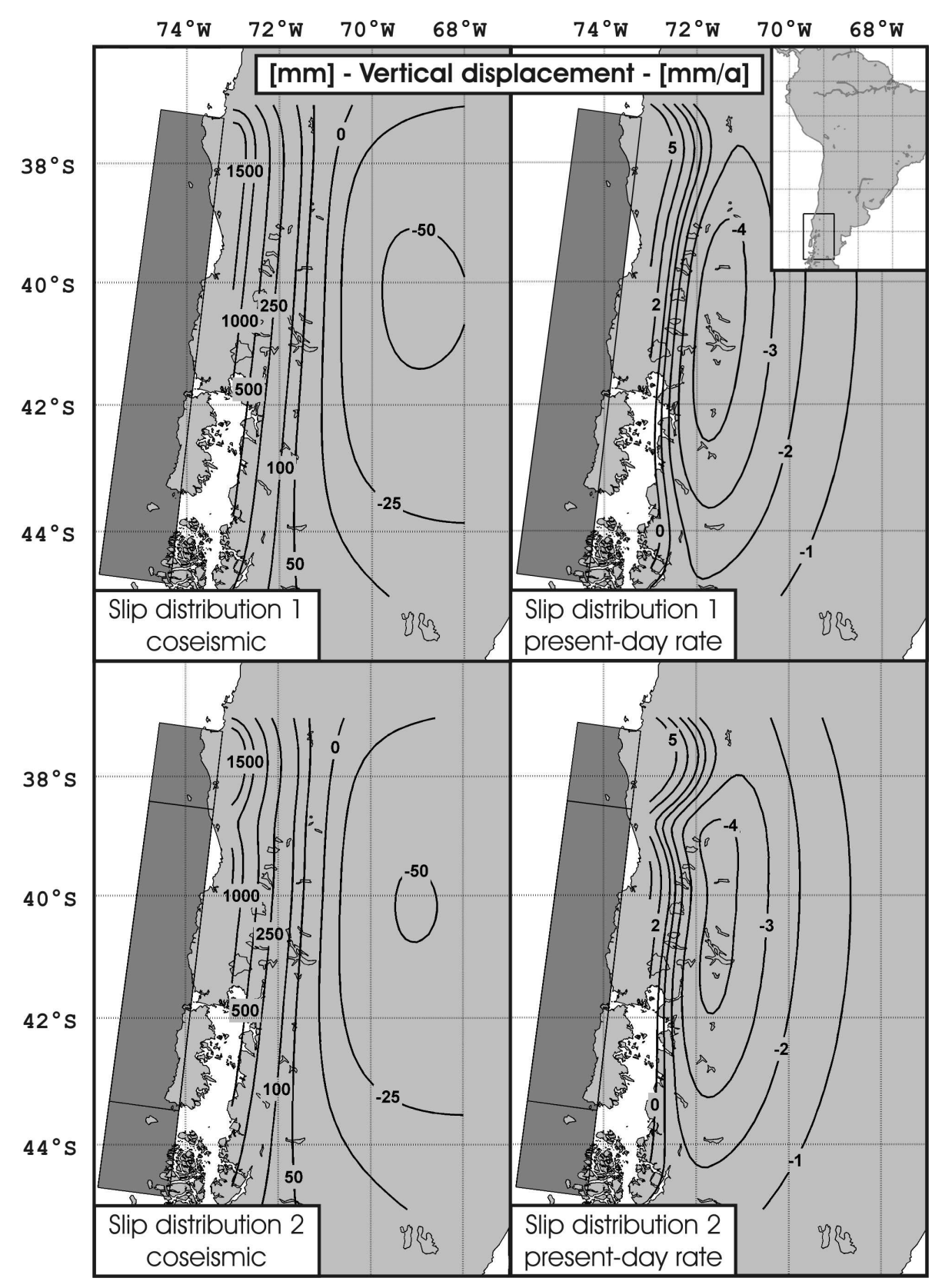

Fig. 5. Same as Fig. 4 but for the vertical displacements (downwards positive). 


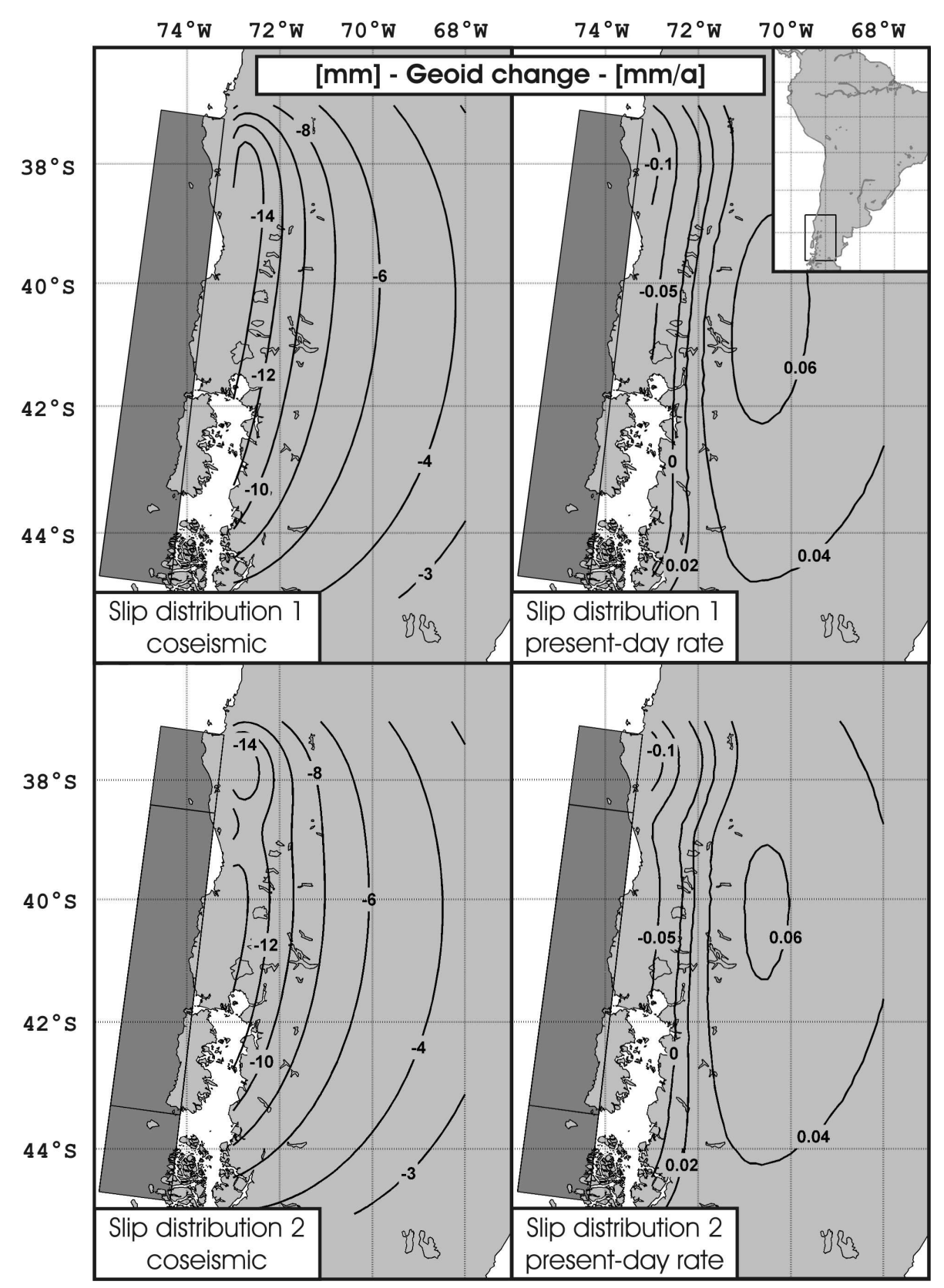

Fig. 6. Same as Fig. 4 but for the geoid changes. 


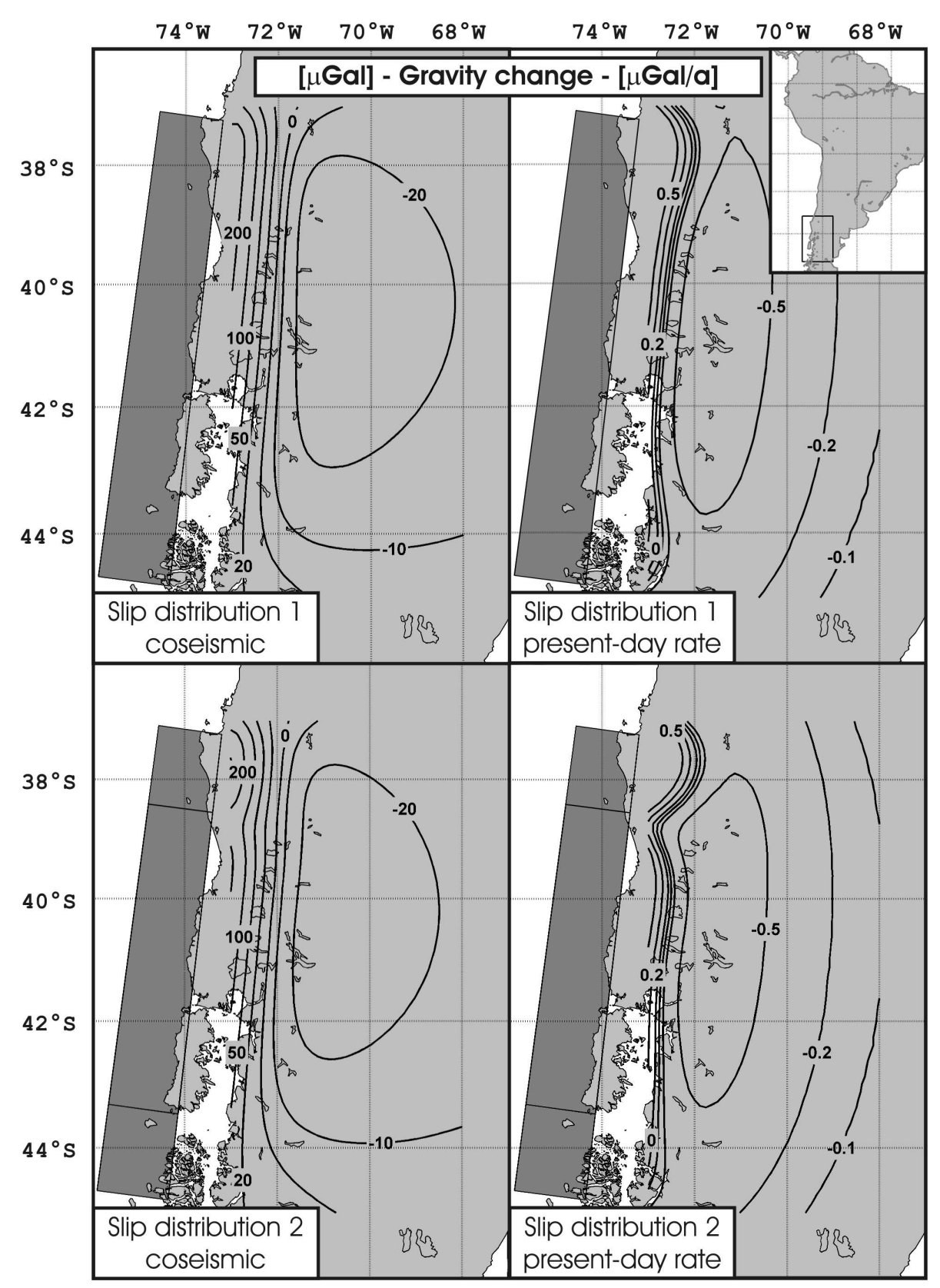

Fig. 7. Same as Fig. 4 but for the gravity changes. Note that here the gravity change is defined by the change measured by a gravimeter, resulting from the interior density change and the vertical motion of the gravimeter ("free air correction"). 


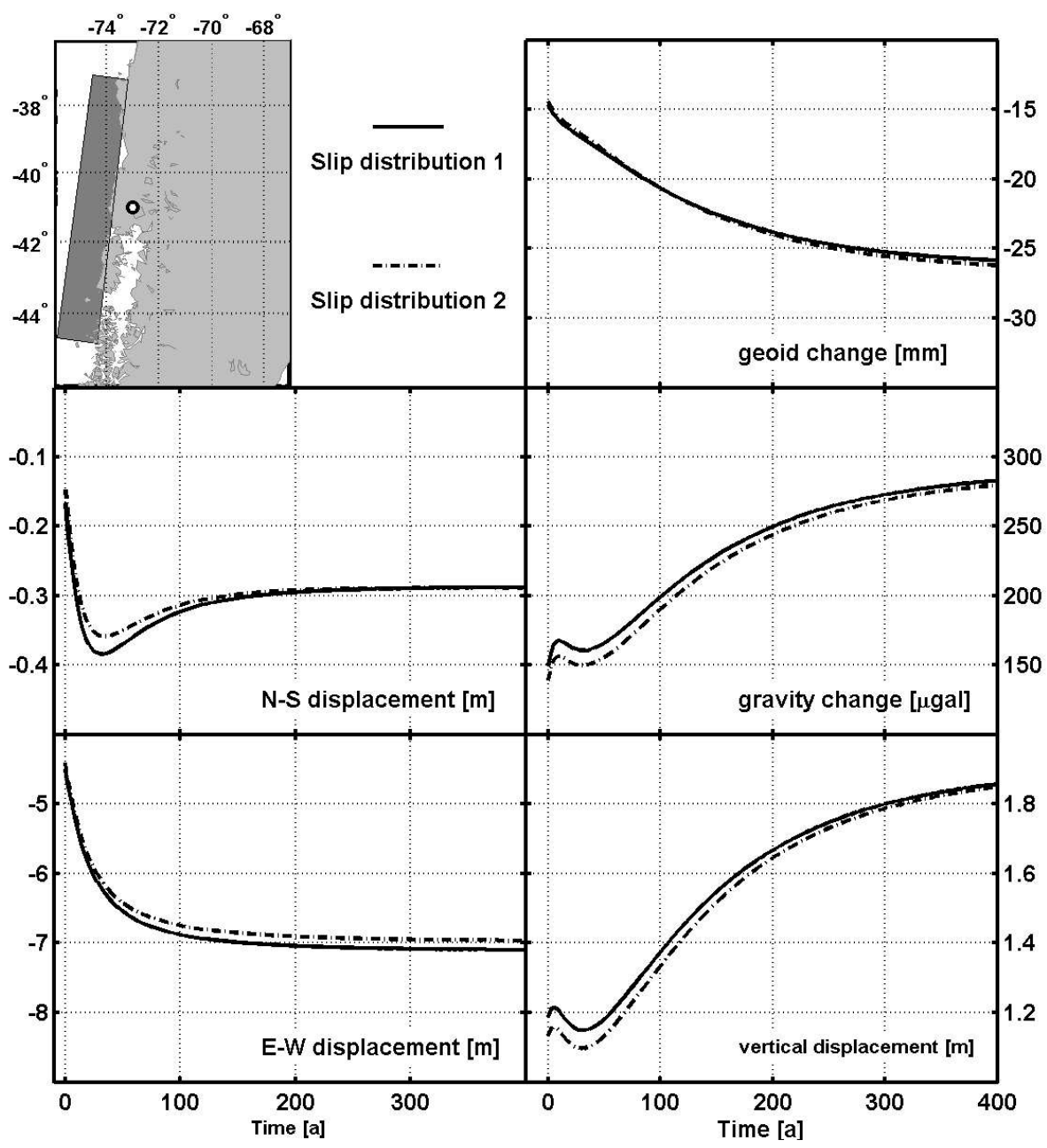

Fig. 8. Time histories of the co- and post-seismic changes for the position at $73^{\circ} \mathrm{W}$ and $41^{\circ} S$ and for the two slip distributions. The local Cartesian coordinate system is used: The N-S component is positive northwards, the E-W component is positive eastwards, and the vertical component is positive downwards. 